\title{
ISSN: 0031-7985
}

0031-7985 is the Print ISSN of Philologus.

About Philologus

\begin{tabular}{|c|c|}
\hline Abbreviation & Philologus \\
\hline \multirow[t]{2}{*}{ ISSN } & $0031-7985$ (Print) \\
\hline & 0031-7985 (Linking) \\
\hline Publisher & Berlin [etc.] Akademie-Verlag [etc.] \\
\hline Language & English, German \\
\hline
\end{tabular}




\title{
PHILOLOGUS
}

Zeitschrift für antike Literatur und ihre Rezeption

\author{
HERAUSGEBER \\ Widu-Wolfgang Ehlers (Berlin), Fritz Jürß (Berlin), \\ Wolfgang Rösler (Berlin), Peter Lebrecht Schmidt (Konstanz), \\ Bernd Seidensticker (Berlin) \\ REDAKTIONSBEIRAT \\ István Borzsák (Budapest), William M. Calder III (Urbana), \\ Rismag Gordeziani (Tblisi), Carlos Lévy (Paris), \\ Benedetto Marzullo (Roma), Adelina Piatkowski (Bucureşti), \\ P. H. Schrijvers (Leiden), Heikki Solin (Helsinki), \\ Nigel G. Wilson (Oxford)
}

Geschäftsführende Herausgeber des Bandes 151:

Widu-Wolfgang Ehlers und Bernd Seidensticker

\section{BAND 151 \\ $2007 \cdot$ Heft 1}

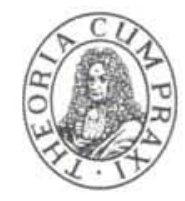

Akademie Verlag 
Die Zeitschrift PHILOLOGUS dient der Erforschung des griechisch-römischen Altertums in seinen geistig-kulturelien Formen und deren Wirkungsgeschichte. Die philologischen Untersuchungen betreffen sprachlich-begriffliche und textkritische Probleme ebenso wie allgemeine Fragen der Literatur, Religion, Philosophie und Wissenschaft der Antike.

Bezugsmöglichkeiten

Bitte richten Sie Ihre Bestellungen an

Oldenbourg Verlagsgruppe, Zeitschriftenservice, Postfach 8013 60, D-81613 München,

Telefon: (0 89) 450512 29/3 99, Telefax: (0 89) 4505 13 33, E-Mail: vertrieb-zs@oldenbourg.de.

\section{Zeitschrift „PHILOLOGUS“}

Herausgeber: Prof. Dr. W. W. Ehlers, Prof. Dr. F. Jürß, Prof. Dr. W. Rösler, Prof. Dr. P. L. Schmidt, Prof. Dr. B. Seidensticker.

Anschrift der geschäftsführenden Herausgeber: Prof. Dr. W. W. Ehlers, Prof. Dr. B. Seidensticker, Freie Universität Berlin, Seminar für Klassische Philologie, Ehrenbergstr. 35, D-14195 Berlin. Telefon: (0 30) $83852211 / 4293$, Telefax: (0 30) 83852767.

Verlag: Akademie Verlag GmbH, Palisadenstr. 40, D-10243 Berlin; Telefon: (0 30) 422006 40, Telefax: (030) 422006 57; www.akademie-verlag.de.

Geschäftsführer: Johannes Oldenbourg.

Verlagsleitung: Dr. Sabine Cofalla.

Anzeigenannahme: Christina Gericke, Akademie Verlag GmbH, Telefon: (0 30) 42 2006 40; Telefax: (0 30) 422006 57, E-Mail: gericke@akademie-verlag.de.

Satz: WERKSATZ Schmidt \& Schulz GmbH, D-06773 Gräfenhainichen.

Druck und buchbinderische Weiterverarbeitung: MB Medienhaus Berlin GmbH, D-12107 Berlin.

Erscheinungsweise: Die Zeitschrift erscheint jährlich in einem Band mit 2 Heften. Jahresbezugspreis 2007: Inland und Ausland $€ 214,00$. Einzelheft $€ 116,00$. Vorzugspreis für Mitglieder von Fachgesellschaften und Studenten $€ 90,00$ jeweils zuzüglich Versandkosten.

Das Abonnement verlängert sich jeweils um ein weiteres Jahr, falls es nicht 8 Wochen vor Ablauf eines Kalenderjahres gekündigt wird.

Authorization to photocopy items for internal or personal use, or the internal or personal use of specific clients, is granted by Akademie Verlag GmbH, provided that the base fee of US $\$ 10.00$ per copy, plus US $\$ 0.25$ per page is paid directly to copyright Clearance Center, 27 Congress Street, SALEM, MA 01970) USA. For those organizations that have been granted a photocopy license by CCC, a separate system of payment has been arranged.

The fee code for users of the Transactional Reporting Service is: 0031-7985/2007 \$10.00+0.25.

Urheberrecht: Alle Rechte vorbehalten, insbesondere die der Übersetzung. Kein Teil dieser Zeitschrift darf in irgendeiner Form - durch Fotokopie, Mikrofilm oder irgendein anderes Verfahren - ohne schriftliche Genehmigung des Verlages reproduziert oder in eine von Maschinen, insbesondere von Datenverarbeitungsanlagen verwendbare Sprache übertragen oder übersetzt werden.

Gedruckt auf alterungsbeständigem Papier.

C 2007 by Akademie Verlag GmbH. Printed in the Federal Republic of Germany. 


\begin{tabular}{|l|l|l|l|l|}
\hline Philologus & 151 & 2007 & 1 & $3-16$ \\
\hline
\end{tabular}

\section{LaUra Carrara}

\section{IL PROCESSO AREOPAGITICO DI ORESTE: LE EUMENIDI DI ESCHILO E LA TRADIZIONE ATTICA*}

Mettendo a confronto le innovazioni attuate da Eschilo nelle tre tragedie che compongono l'Orestea, già Albin Lesky aveva con ragione concluso: „Am größten ist die Freiheit des Dichters der Tradition gegenüber in den Eumeniden". ${ }^{1}$ Il problema di una analisi puntuale di questa libertà compositiva è però singolarmente rimasto ai margini dell'attenzione degli studiosi: dovendo valutare l'impronta personale lasciata da Eschilo sulla tradizione leggendaria precedente a proposito della soluzione della vicenda di Oreste, la critica si è spesso limitata ad accettare la tesi esposta per la prima volta, a mia conoscenza, da K. O. Müller: „So konnte also die Sage von Orestes Lossprechung durch den Areopag auch keinen geschichtskundigen Athener befremden. (...) auch erzählt sein Zeitgenoss Hellanikos, wie nicht blos Orestes, sondern viele andre Heroen, ja Götter, vor ihm in diesem Gericht ihr Urtheil empfangen hätten". ${ }^{2}$

Si possono muovere varie obiezioni a questa ipotesi, sulla base di alcune considerazioni di cui il Müller sembra non essere stato il solo a sottovalutare la portata. La sua interpretazione infatti, come accennato sopra, riscuote ancor oggi il consenso generale e si è imposta come communis opinio sull'argomento. Ma si può proporre un approccio diverso: fu Eschilo a ideare la risoluzione della vicenda di Oreste attraverso il processo areopagitico (con giudici umani) e non esisteva nessuna tradizione locale attica che raccontasse di Oreste processato ad Atene prima del 458 a.C.

A fondamento della sua tesi il Müller pone un frammento dell'attidografo Ellanico

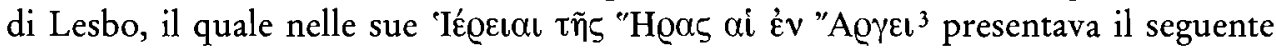
resoconto (fr. 169 Fowler ${ }^{4}$ ):

* Sono grata al prof. G.W. Most per aver promosso la pubblicazione di questo articolo e averne seguito la stesura e al prof. E. Medda per i suoi utili consigli. Desidero ringraziare inoltre i miei amici e colleghi Chiara Meccariello, Eva Falaschi, Arianna Marelli e Stefano Rinaldi che hanno accompagnato il mio lavoro con il loro affettuoso sostegno. Questo articolo è dedicato a Chiara, senza la quale nemmeno una riga avrebbe potuto essere scritta. Degli errori rimasti e delle scelte finali resto unica responsabile.

1 A. Lesky (1939) 980.

2 Müller (1833) 157. Analoga conclusione in Wecklein (1888) 9-10, Wilamowitz (1904) 243-245 e (1914) 189, Robert (1926) 1321-1322, Croiset (1928) 237, Lesky (1931) 190-214 e (1939) 979-982, Mazon (1955) I-XIX, Quincey (1964) 198-199, Sommerstein (1989) 5.

3 Per le quali la data di composizione indicata da Jacoby (1954 a) 6-9 è il $420 \mathrm{ca}$.

${ }^{4}$ FGHist 323 a F $22=\Sigma$ Eu. Or. v. 1648. 


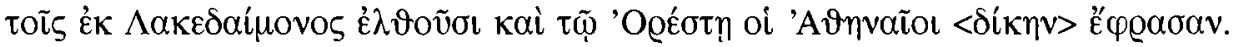

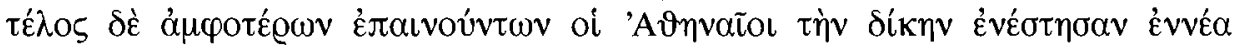

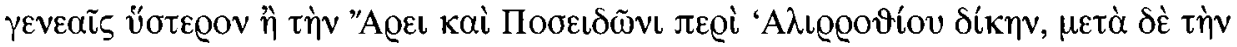

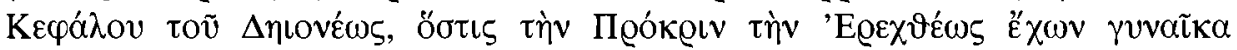

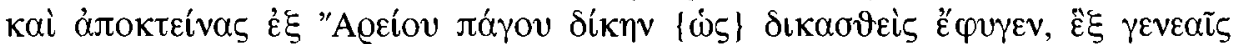

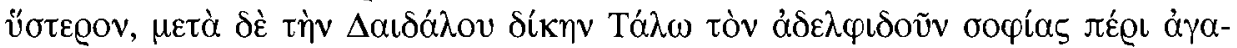

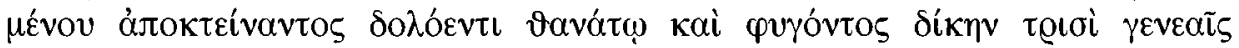

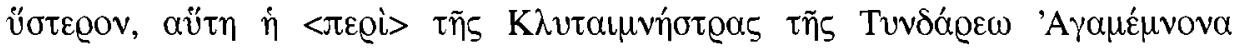

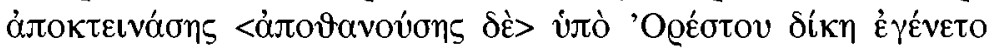

Il problema posto da questo testo consiste nell'accertare in quale misura (che significa per quali processi) esso rispecchi la „versione dei fatti“ diffusa ad Atene prima di Eschilo. I primi tre processi di cui Ellanico dà notizia, quelli a carico di Ares, Cefalo e Dedalo, datano senz'altro a un tempo antico, un tempo in cui l'Areopago era l'unico tribunale per le cause di sangue. Se anche si ammette che le Eumenidi avessero fatto ulteriormente crescere l'attenzione sull'Areopago, è incredibile che si siano inventate dopo il $458 \mathrm{a}$. C. delle storie che correvano così palesemente contro la pratica giudiziaria del V secolo: in Ellanico gli accusati di póvos ảkov́otos (Cefalo) o díkotos (Ares) compaiono davanti all'Areopago, mentre nel V secolo queste tipologie di delitto erano rispettivamente di competenza del Palladio e del Delfinio ${ }^{5}$. Per i primi tre casi dunque il valore della testimonianza di Ellanico in riferimento ad un tempo a lui anteriore di circa due secoli (prima dei vóuot potvıкoí) non deve essere messo in discussione. $\mathrm{Ma}$ è lecito credere che anche per il caso di Oreste le parole di Ellanico riflettano uno stato di cose così antico, cioè che già nella tradizione attica pre-eschilea si conosceva un processo di Oreste celebrato, come quarto della serie, sull'Areopago?

L'argumentum ex silentio è ben lontano dall'essere risolutivo: il fatto che - oltre ad Ellanico - non esista nessun'altra chiara testimonianza sicuramente anteriore ad Eschilo (o riferibile a un tempo precedente la sua Orestea) che parli di un processo di Oreste può dipendere, in linea teorica, soltanto dallo stato lacunoso della nostra documentazione. Sempre in linea teorica, non è quindi illegittimo il tentativo - operato dal Müller in avanti - di rintracciare i disiecta membra di questa (ipotetica) tradizione pre-eschilea e comporli poi in un quadro coerente.

In particolare, tre notizie riferentisi a un periodo anteriore al 458 a. C. sarebbero per noi pienamente intelligibili - così si è supposto - solo se si postula come sfondo su cui proiettarle un già esistente processo areopagitico di Oreste.

${ }^{5}$ Si rimanda in proposito anche a Wallace (1985) 9-10 che precisa e accetta questa argomentazione, già sviluppata da Jacoby (1954b) 22 n. 22. C’è un secondo motivo che depone a favore dell'esistenza della storia di Ares (e di Cefalo e Dedalo) prima di Eschilo: è per far dimenticare la già diffusa etimologia di Areopago legata al nome di Ares che Eschilo di proposito etimologizza diversamente in Eum. 681-693 (cf. infra 14 con n. 31). 
La prima traccia che tale tradizione attica avrebbe lasciato precedentemente e al di fuori dell'opera storica di Ellanico si trova nello scolio ad Eur. Or. 1645 che riporta, tra le altre, la versione di Ferecide sulla fine della vicenda di Oreste (fr. 135 Fowler):

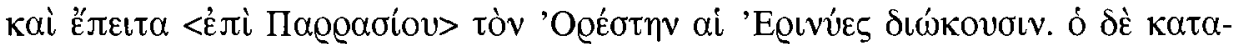

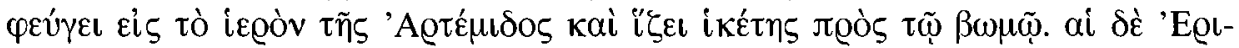

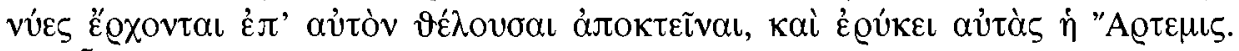

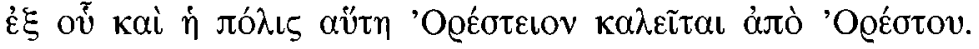

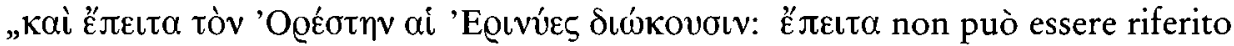
ad altro se non al processo sull'Areopago“. Così, nel 1923, Felix Jacoby ${ }^{6}$. In altri termini, già nel primo quarto del V secolo, Ferecide conoscerebbe una versione secondo cui le Erinni non desistono dall'inseguire Oreste, in fuga verso l'Arcadia, nemmeno dopo che il voto areopagitico ha decretato l'assoluzione del matricida. La

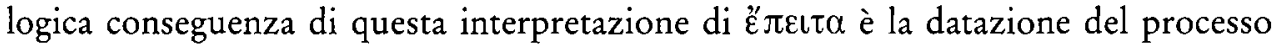
areopagitico di Oreste a un periodo pre-eschileo. Ma lo stesso Jacoby nella riedizione dei frammenti di Ellanico ${ }^{7}$, discostandosi dalla posizione precedentemente assunta, nega che ع̈ $\pi \varepsilon \imath \tau \alpha$ sia da intendere „dopo il processo dell'Areopago“. Tuttavia, il nuovo argomento addotto si rivela fragile: si limita a constatare che Ferecide, contemporaneo più anziano di Eschilo, non poteva riferirsi ad un processo che non era stato ancora inventato e si chiude in un circolo vizioso: per dimostrare che Ferecide, il cui scritto è anteriore al 458 a.C., non può conoscere il processo areopagitico di Oreste si afferma che questo è stato inventato da Eschilo, e per sostenere tale ipotesi si adduce come prova l'ignoranza del processo da parte del più anziano Ferecide.

Senza inoltrarsi sull'incerto terreno della cronologia relativa, Jacoby avrebbe potuto appoggiarsi unicamente alla sua seconda, penetrante, osservazione: ammesso che la tradizione attica conoscesse già prima di Eschilo il mito del processo di Oreste, la forma assunta da questo mito non poteva essere quella desumibile dal frammento di Ferecide letto secondo l'interpretazione del 1923 , perché qui il verdetto ateniese non è per nulla sufficiente a bloccare la persecuzione delle Erinni. Una tale conclusione della vicenda di Oreste sarebbe servita molto male a uno dei fini per cui il giudizio areopagitico è stato creato - a prescindere dalla data di questa creazione: mostrare che solo Atene riusciva a decidere del destino dell'ultimo protagonista della saga mitica più celebre e più complessa di tutta la Grecia 8 .

6 Jacoby (1923) 424. Successivamente lo stesso Jacoby, dopo aver indotto all'errore anche Lesky (1939) 985 avrà l'intuizione giusta suggerendo che fu Eschilo il primo a portare Oreste davanti all'Areopago (vd. infra).

7 Jacoby (1954b) 24-25 n. 24.

8 Merita forse di essere ricordato che nemmeno gli scrittori di storia locale del Peloponneso mettevano in

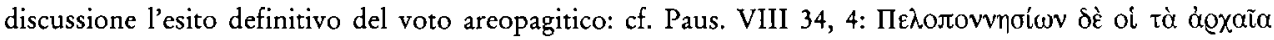

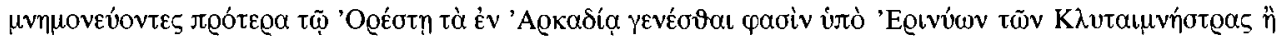

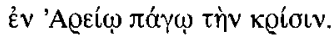


Una deroga a questo principio pare essere la Ifigenia in Tauride di Euripide ai vv. 970-975:

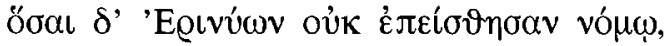

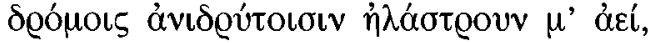

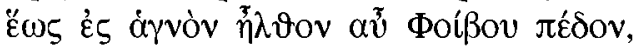

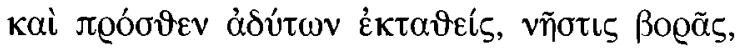

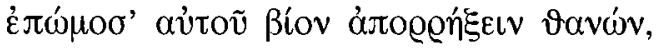

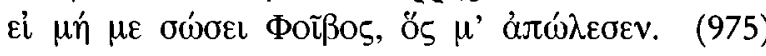

In questo passo euripideo è chiaramente detto quel che è solo congetturale in relazione al passo di Ferecide: le Erinni (o meglio, alcune di esse) non sono placate dal voto areopagitico e continuano a inseguire Oreste. È possibile che Euripide stia qui recuperando una antica versione del mito simile - almeno nelle linee generali - a quella che Jacoby supponeva nel 1923 per Ferecide fr. 135 Fowler. Ma questa resta una possibilità solo teorica, perché nell' Ifigenia in Tauride „die ganze Fabel des Dramas ist seine [scil. di Euripide] freie Erfindung ". ${ }^{9}$ L'innovativa scelta di Euripide è strettamente funzionale al suo desiderio di dare un aition al culto di Artemide a Brauron, di cui Ifigenia - così si raccontava - era sacerdotessa, collegando tale culto alla permanenza di Ifigenia in Tauride. In altri termini, è per motivare il viaggio di Oreste in Tauride - alla fine del quale (Iph. T. 1438-1441) sarà fondato il culto di Brauron - che Euripide non esita a rendere inefficace il voto dell'Areopago.

La posizione di Euripide nei confronti del mito è quindi del tutto particolare, e non invalida l'analisi fino ad ora condotta riguardo a Ferecide. Non si può qui stabilire con certezza quale avvenimento precedesse la fuga di Oreste ad Oresteion secondo questo attidografo ${ }^{10}$. Qui può non essere stato inutile aver richiamato la difficoltà che sorge

9 Wilamowitz (1883) 254: „Die Vereinigung beider [scil. dei due ruoli di Ifigenia, in Tauride e a Brauron] ist ein Werk seiner [scil. di Euripide] eigenen Genialität, von einem Erfolge, welcher freilich beweist, was einem Dichterwillen gelingen kann, wenn er im Sinne seiner Zeit die Heldensage fortsetzt." Analogamente giudica Platnauer (1967) xi-xii, che qualifica l'operazione euripidea come scarto dalla versione eschilea (non come recupero di una tradizione più antica): ,it is likely that Euripides introduces Orestes into the story [scil. di Ifigenia a Brauron e in Tauride]." Cf. anche Robert (1926) 1327-1328: “Der Dichter knüpfte an den Kult der Artemis von Brauron an."

10 Secondo Jacoby (1954b) 24 n. 24, è stato forse lo scoliasta a intendere ě [ $\pi \varepsilon \iota \tau \alpha$ come „dopo il processo" e ha cirato Ferecide perché presentava a suo parere un ordine degli avvenimenti inverso rispetto a quello del passo dell' Oreste euripideo che stava commentando, dove l'esilio in terra straniera (Arcadia) precede il processo ateniese (mentre in Ferecide il processo precederebbe l'esilio). Questa ricostruzione è artificiosa: si basa sul presupposto che lo scoliaste potesse leggere solo queste poche parole di Ferecide e le facesse automaticamente rientrare nelle sue categorie mentali „eschilee“. C'è un modo più semplice e immediato di intendere lo

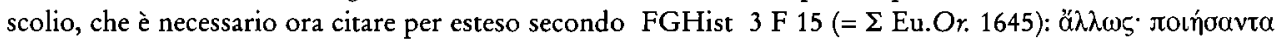

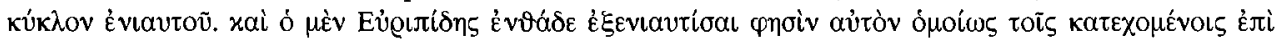

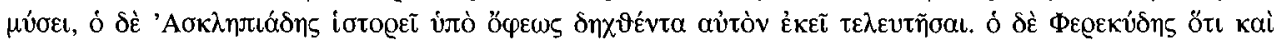


presumendo che il discrimen costituito da é $\pi \varepsilon \iota \tau \alpha$ sia l'azione legale: un finale di mito organizzato secondo la sequenza temporale supposta per Ferecide (processo e a seguire reiterata persecuzione delle Erinni) non avrebbe soddisfatto lo scopo per cui il mito stesso era stato ideato.

La seconda tradizione pre-eschilea di cui si è ritenuta impossibile la nascita senza la preliminare esistenza del processo di Oreste è costituita dal mito di fondazione del rituale della festa delle Coe. Esso trova la sua prima, certa espressione letteraria nell'Ifigenia in Tauride euripidea ai vv. 945-96011.

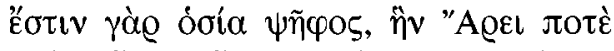

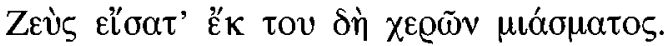

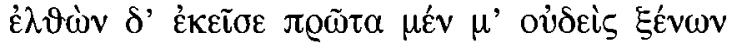

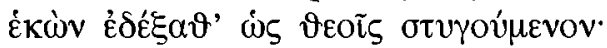

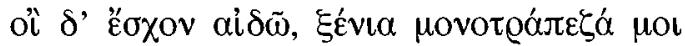

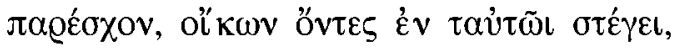

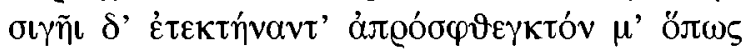

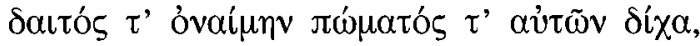

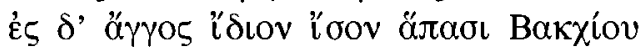

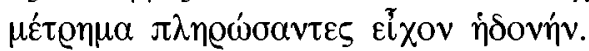

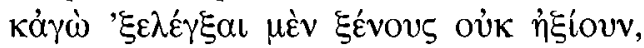

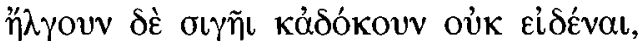

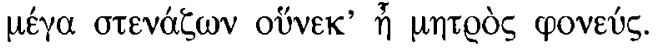

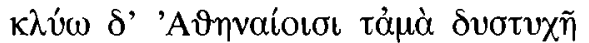

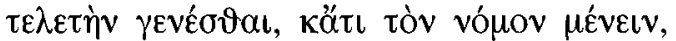

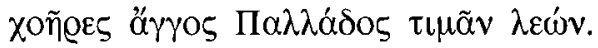

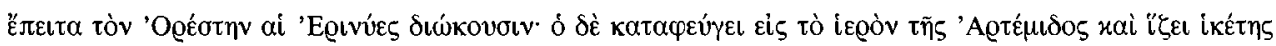

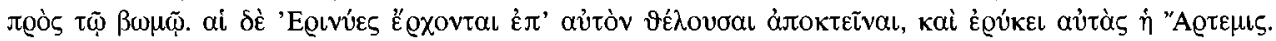

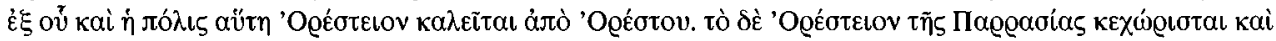

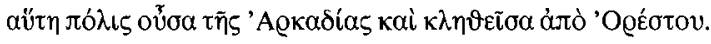

Lo scoliaste sta raccogliendo varie versioni sulle vicende occorse ad Oreste in Parrasia, che è il focus della

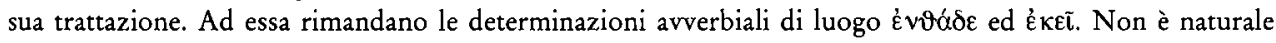
pensare che il referente dell'avverbio di tempo ëjeıt $\alpha$ sia sempre il medesimo, cioè, ancora una volta, la pianura Parrasia? Si potrebbe quindi così parafrasare ed esplicitare: „Secondo Ferecide, le Erinni inseguono Oreste anche dopo il suo esilio in Parrasia, perciò egli è costretto a cercare scampo ad Oresteo." Non sarà ozioso localizzare con esattezza Oresteo: cf., oltre al finale di $\Sigma$ Eu. Or. 1645, anche Willink (1986) 354 „on the

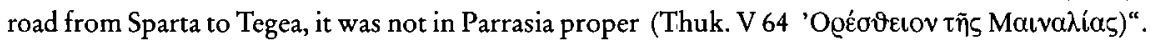

${ }_{11}$ Elenco delle altre fonti antiche in Burkert (1996) 246 n. 31. Burkert riferisce l'idea che l'aition delle Coe

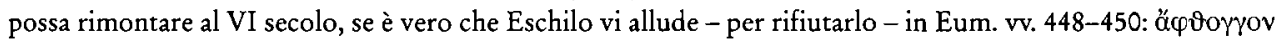

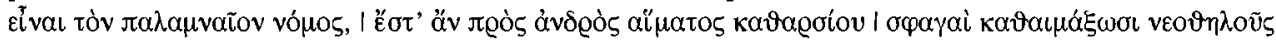
ßotoṽ. L'esistenza di una allusione in questi versi è dubbia; ci sono altri, più cogenti motivi (cf. n. 12) per retrodatare l'aition a un tempo precedente l' Iph. T. Qualsiasi datazione si accolga, essa non influenza l'indagine qui svolta. 
L'aition delle Coe istituisce un collegamento tra il destino di Oreste matricida e la città di Atene. Si è ipotizzato che solo l'esistenza di una tradizione più antica che già connetteva Oreste e Atene avrebbe potuto permettere questo collegamento, fornendo all'aition delle Coe il terreno adatto su cui innestarsi ${ }^{12}$ : „In any case it is unlikely that anyone would have thought of accounting thus for the rules of the Choes festival if it were not already an established mythic 'fact' that Orestes had come to Athens after his matricide; and the Choes myth is thus probably, so to speak, parasitic on the only other tale we know of that brings Orestes to Athens at that stage of his career - the tale of his being tried for murder on the Areopagus" 13 .

Sommerstein descrive una situazione in cui il processo areopagitico di Oreste funziona da "trampolino di lancio" per la creazione della storia delle Coe, ma non spiega per quale motivo tale "trampolino" debba necessariamente essere il processo areopagitico, e non un' altra tradizione ateniese su Oreste (quella odissiaca, per cui cf. nn. 14 e 17). Ma la difficoltà più grave del ragionamento di Sommerstein risiede altrove, nel presupposto stesso, non sufficientemente fondato, che questo aition abbia bisogno di appoggiarsi a una preesistente connessione Oreste - Atene. La storia delle Coe era originariamente indipendente, allo stesso modo di tanti altri aitia inventati con l'unico proposito di spiegare un rito. L'aition delle Coe può essere accostato come cronologia e come genesi a quel gruppo di affermazioni, nate tra VII e VI secolo, che Atene cominciò a mettere in campo per conquistare un ruolo di primo piano nelle vicende della grande saga della guerra di Troia, ruolo che i poemi omerici non le avevano riservato. Così si inventarono tra gli altri ${ }^{14}$ :

12 C’è una soluzione più radicale al problema posto dall'aition delle Coe che ugualmente non obbliga a immaginare come già esistente il processo areopagitico: è sufficiente posticipare la nascita dell'aition in età post-eschilea, facendone un'invenzione euripidea, e individuare nella stessa Orestea l'antecedente di questa (nuova) vicissitudine ateniese di Oreste. Questa ipotesi crea più problemi di quanti ne risolva. Se l'aition delle Coe fosse nato con la $I p h$. $T$., da dove provengono i particolari cultuali (le corone, i riti al santuario delle Paludi) che non si trovano in Euripide, ma, per es., in Fanodemo (FGHist $325 \mathrm{~F} \mathrm{11)}$ ? E verosimile che li abbia introdotti per primo l'attidografo? Inoltre, Euripide parla (Iph. T. 955) degli ̧́́vou di Oreste senza spiegare chi siano. Gli spettatori non avrebbero potuto trattare come un „fatto“ mitico in sé comprensibile questi $\xi$ ḱvol se non riallacciandosi a una tradizione che conosceva l'ospitalità offerta da Atene a Oreste. Euripide qui può chiedere al suo pubblico di supplire mentalmente le informazioni da lui omesse solo perché vi era un sapere comune da cui tali informazioni potevano essere attinte (sapere confluito, per es., in Plut. Symp. 643 a da cui veniamo a sapere che questi ospiti sono i Demofontidi, cioè re Demofonte, i suoi familiari, il suo popolo:

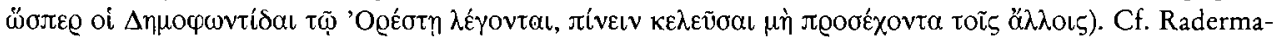
cher (1903) 133-140.

13 Sommerstein (1989) 3-4.

14 Forse proprio in quest'ambito si genera - e quindi si spiega - anche la misteriosa menzione di Atene nell'Odissea che costituisce per noi la prima testimonianza dell'esistenza di un legame tra Oreste e Atene (Od.

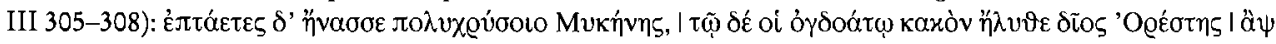

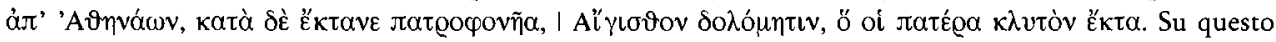
passo già i commentatori antichi non riuscivano a gettare luce. Chi ha riconosciuto la difficoltà, ha tentato di risolverla in diversi modi, nessuno soddisfacente. Zenodoto cambia in ảjó $\Phi \omega \kappa \eta ́ \omega v$, Aristarco - in modo

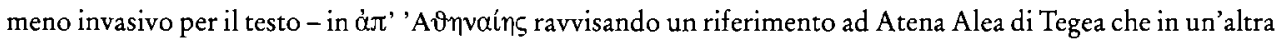
versione antica (a quanto pare poi scomparsa) doveva proteggere Oreste. Sicuramente gli eruditi alessandrini 
1) un sacrificio di Ifigenia a Brauron e non in Aulide (Phan. FGHist 325 F 14, $\sum$ Aristoph. Lys. v. 645),

2) la presenza di Demofonte e Acamante, figli di Teseo, sotto le mura di Troia ${ }^{15}$,

3) gli aitia del tribunale Palladio, con protagonisti Agamennone e Diomede ${ }^{16}$.

Questi ultimi due aitia rivestono un interesse maggiore perché si lasciano datare con certezza: sono senz'altro posteriori alla legislazione di Dracone che per prima distinse i vari tipi di vómol pouvıkó e i relativi tribunali. A proposito di questi aitia, nessuno ha sostenuto che per far sbarcare Diomede o Agamennone in Attica fosse necessario un preesistente collegamento tra questi eroi e questa regione, e non c'è motivo perché un discorso analogo non possa valere anche per Oreste ospite di Demofonte nel secondo giorno delle Antesterie ${ }^{17}$.

L'aition delle Coe è dunque "in sé" sussistente e spiegabile all'interno dell'universo del VI sec., religioso (se comprendeva la purificazione ${ }^{18}$ ) e soprattutto politico ( $\mathrm{fa}$

non conoscevano nessuna opera di età arcaica o classica che connettesse Oreste e Atene in modo simile al passo odissiaco, altrimenti non avrebbero perso l'occasione di citarla a chiarimento dell'enigmatico verso (devo questa osservazione al prof. G. W. Most).

15 Cf. Barrett (1964) 2-3, Radermacher (1903) 133-140.

16 Per Agamennone, derubato del Palladio da Demofonte, il quale deve subire il processo per aver fatto strage dei compagni dell'Atride cf. Kleid. FGHist 323 F 20.

Per Diomede, sbarcato in Attica e assalito per errore da Demofonte - che subirà il processo per il sangue

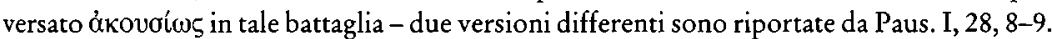

17 Jacoby (1954b) $28 \mathrm{n} .28$ avanza quella che è destinata a restare una ipotesi inverificabile e, per ammissione dello studioso stesso, non necessaria. Se si vuole restare ancorati all'idea che il mito delle Coe abbia bisogno di una "base“, tale base può essere $O d$. III 307: il fatto che Oreste venne da Atene per compiere la vendetta sulla madre e su Egisto implica che avesse lì trovato l'ospitalità del re Demofonte per cui era del tutto

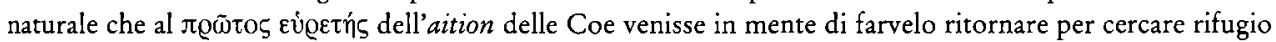
dopo il matricidio.

${ }_{18}$ Che il mito di fondazione si concludesse con una purificazione ad opera del re Demofonte è possibile:

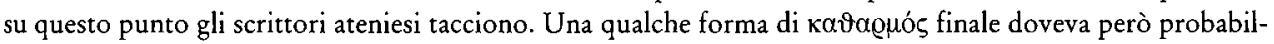
mente esistere nel mito perché non si poteva lasciare Oreste in stato di impurità. Notiamo per incidens che ancora per Eschilo la purificazione delfica costituisce un passaggio obbligato: cf. Robert (1926) 1324: „Die delphische Sage hat Aischylos in seinen Eumeniden mit der attischen zu verbinden gewußt“. L'aition delle Coe poteva (doveva?) quindi terminare in maniera simile a una storia di Trezene tramandataci da Paus. II, 31, 8:

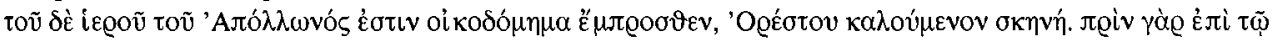

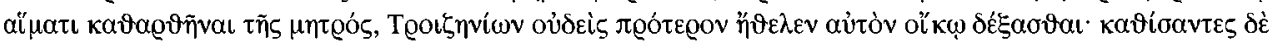

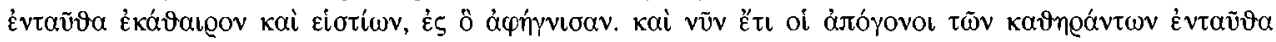

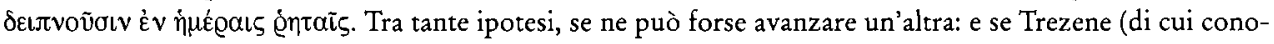
sciamo gli stretti legami con Atene, cf. Barrett (1964) 2-3) rispecchiasse qui, per una sorta di applicazione della "norma dell'area laterale“, una fase della leggenda ereditata dalla potente vicina, e da quest'ultima non conservata? Se si riconosce un minimo di verosimiglianza a questa ipotesi, si può ricordare che la presenza della procedura di purificazione risponde a una esigenza propria della mentalità religiosa di fine VII-VI secolo. Solo alla fine del VII e poi nel VI sec. - non prima - la purificazione degli assassini diviene per le comunità un grosso problema che può trovare soluzione solo su suolo straniero: questo è il caso dell'argivo Oreste ad Atene. Cf. Wallace (1985) 29-30, Parker (1983) 114-118: „It has often been noted that there is no mention of pollution in the surviving portions of Draco's law and other authors of the period who have much to say 
parte della volontà ateniese di acquistare una certa preminenza nella saga troiana) e non c'è ragione di supporre che sia stata "generato“ da una ormai affermata leggenda sul voto dell'A reopago.

La terza ed ultima notizia che si è voluta leggere ${ }^{19}$ alla luce di una già esistente soluzione giudiziaria pre-eschilea coinvolge la composizione del collegio giudicante Oreste come appare da Eur. Or. 1650-1652 ${ }^{20}$ :

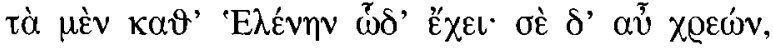

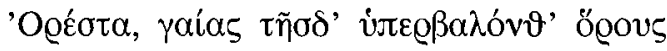

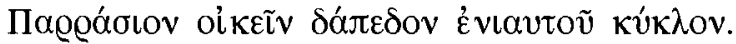

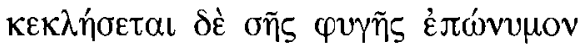

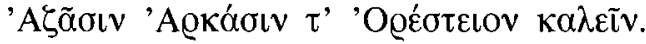

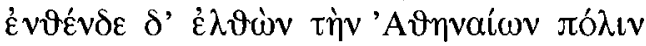

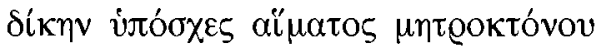

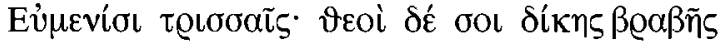

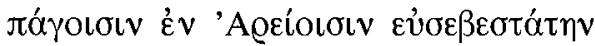

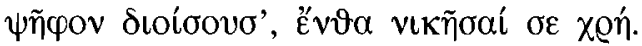

Dopo aver annunciato la divinizzazione di Elena, Apollo predice ad Oreste il suo destino: egli dovrà scontare un anno di esilio nella pianura Parrasia, poi recarsi ad Atene, dove i suoi giudici saranno i Dodici Dei, che lo assolveranno dall'accusa di matricidio mossagli dalle tre Eumenidi.

Willink e Di Benedetto ${ }^{21}$ notano che nell'Oreste i Dodici Dei rivestono il ruolo di giudici in conformità con l'antica saga attica, cui Euripide si starebbe qui richiamando: l'innovazione di Eschilo sarebbe quindi consistita solo nel rendere i giudici umani, non nell'inventare il processo.

about justice and the welfare of community (Hesiod, Archilocus, Alcaeus, Solon) do not seem to be haunted by the spectre of the unpunished murderer lurking in its. midst“ (p. 116); „His [scil. dell'assassino] taint, inaffaceable at home, disappears, or at least becomes open to purification, as soon as he reaches foreign soil“

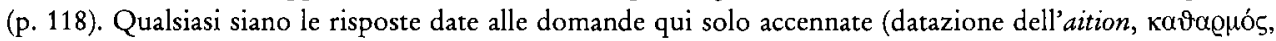
rapporti Atene-Trezene), esse non intaccano la validità dell'assunto principale: l'indipendenza del mito delle Coe e del mito del processo.

${ }^{19}$ La menzione dei Dodici Dei nelle vesti di giudici è l' evidenza addotta più di frequente a sostegno del processo pre-eschileo. La considerano una prova decisiva: Müller (1833) 157, Wecklein (1888) 9, Robert (1926) 1321-1322, Croiset (1928) 237, Lesky (1931) 96, Mazon (1955) VIII, con qualche dubbio Quincey (1964) 198: „They [scil. Euripide e Demostene] indicate a tradition indipendent of, and perhaps earlier than, the Oresteia“.

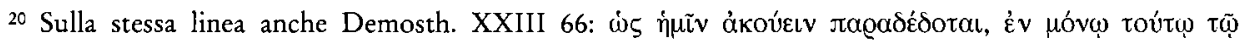

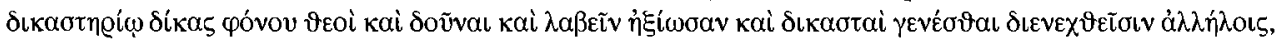

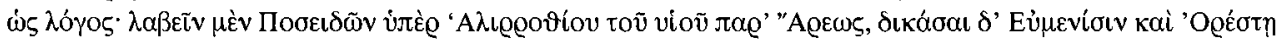

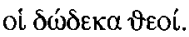

21 Willink (1986) 354-355, Di Benedetto (1965) 299. 
Felix Jacoby ha giustamente ribadito l'inesistenza di questa antica tradizione

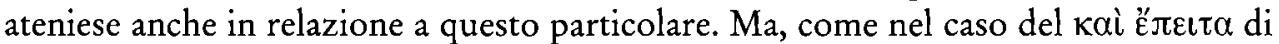
Ferecide, anche qui la sua tesi, corretta, è supportata da un argomento tutt'altro che decisivo: Jacoby si limita infatti ad affermare che i Dodici Dei sono „a later enhancement" all'interno dell' universo mitico dei Greci e confessa di non riuscire a convincersi del fatto che il loro ruolo come giudici sia antico (precedente al $\mathrm{V}$ secolo) ${ }^{22}$. $\mathrm{Ma}$ chi sono allora per lui i giudici di Ares, Cefalo, Dedalo, di cui egli stesso scrive „it is much more probable that the tales date from a time in which the Areopagos was the only court for homicidal trials"? Nessuno potrebbe mettere in dubbio che i giudici di Ares, un dio, dovessero essere a loro volta dei; si veda, oltre al già citato (supra pp. 6-7) Eur. Iph. T. 945-946 (dove la menzione del solo Zeus va intesa per metonimia), anche Eur. El. 1258-1261:

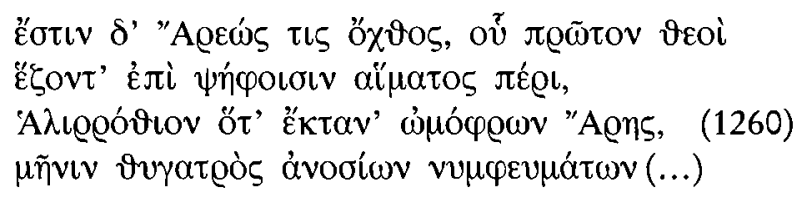

I Dodici Dei cominciarono a esercitare la loro funzione di giudici ben prima degli ultimi decenni del V secolo: nessun altro al di fuori di loro poteva aver svolto questo ruolo nel processo di Ares (e di Cefalo e Dedalo) durante - al minimo - il secolo e mezzo precedente ${ }^{23}$.

I Dodici Dei esistevano dunque come giudici prima di Euripide e anche prima dell'Orestea di Eschilo ${ }^{24}$. Ma ciò non implica che esistessero come giudici di Oreste e certamente non deve essere inventata una saga ad hoc per procurare una fonte antica al passo dell'Oreste euripideo. La menzione dei Dodici Dei nell'Oreste può infatti essere nata dalla logica interna della tragedia nel senso che Euripide non ha potuto evitare di ricorrere al giudizio divino: gli uomini riuniti nell'assemblea di Argo hanno già emesso una sentenza di condanna contro Oreste (vv. 857-858) che solo un tribunale „superiore“ può invalidare. Da qui Euripide può aver avuto l'idea di recuperare, per risolvere l'impasse drammaturgica, l'altro collegio giudicante già deputato nell'immaginario del suo pubblico alle cause di sangue sull'Areopago: scartata la soluzione civica eschilea, non restano che i Dodici Dei, giudici tradizionali trasferiti

22 Jacoby (1954b) 23-24 n. 23.

${ }^{23}$ Jacoby (1954b) 23 n. 23 per mostrare che l'attività positiva dei Dodici Dei nella sfera della storia mitica è praticamente inesistente cita $O$. Weinreich (1977) 833, dove l'autore ammette la presenza esile dei Dodici Dei nel mito greco e scrive: „Ihre [scil. dei Dodici Dei] aktive Rolle beschränkt sich da auf ein Gebiet“. Ma Jacoby avrebbe dovuto avvedersi che questo ambito è esattamente quello giudiziario: essi infatti „geben in Streitigkeiten zwischen zwei Göttern oder bei Blutprozessen als repräsentative Instanz die Entscheidung“.

${ }^{24}$ Si veda ancora Weinreich (1977), dove si legge (772-774) che i Dodici Dei ebbero un culto ad Atene al più tardi con l'altare edificato dai Pisistratidi (cf. Thuk. VI, 54, 6) e che in letteratura „bleibt der Homerische Hermes-Hymnos der älteste literarische Beleg“ (828). 
qui ad un nuovo processo. La loro menzione concentrata in un emistichio (v. 1650) dimostra quanto poco interesse avesse Euripide per uno scioglimento divino della vicenda, una volta che gli uomini (gli Argivi, Tindaro, Menelao - sull'analisi della psicologia umana si incentra la poesia dell'Oreste) si siano rivelati incapaci di elaborare una reazione che metta fine alla catena di violenza nella casa degli Atridi. Il significato della operazione euripidea è stato ben sintetizzato da Biehl: „Die in $1650 \mathrm{ff}$. erhaltene Kritik an der Polis hebt sich deutlich gegenüber der Version des Aischylos ab: dieser hatte in den Eumeniden den Areopagosprozess in den Mythos eingeführt. Euripides mußte deshalb den Prozess als ,attische Tradition“ übernehmen, doch wird das Neue, die Einsetzung der Götter als Richter, gewissermaßen zur „Palinodie“ dessen, was der Vorgänger ausgesagt hatte“25.

Di recente, l'antichità della versione coinvolgente i Dodici Dei è stata nuovamente sostenuta da Sommerstein ${ }^{26}$. A suo avviso, è più probabile una sostituzione dei Dodici Dei con i giudici areopagitici ad opera di Eschilo piuttosto che un cambiamento in direzione opposta poiché come aition della attività giuridica dell'Areopago un giuria umana serve meglio che una divina. Gli Ateniesi, possedendo già nella versione resa celebre da Eschilo un perfetto mito di fondazione per il loro tribunale, l'avrebbero reso meno adatto ai loro scopi se vi avessero introdotto, a fine $\mathrm{V} \mathrm{sec.,} \mathrm{i}$ Dodici Dei. Questi dovevano dunque essere "personaggi“ di una leggenda precedente, imperfetta come aition dell'Areopago, che Eschilo avrebbe migliorato. Un approccio così schematico rischia di travisare il problema, che non va posto in termini di progresso rispetto a una tradizione antica e imperfetta quanto piuttosto in termini di sviluppi diversi rispondenti alle diverse intenzioni degli autori. Non sono stati vagamente e in astratto - gli Ateniesi a inquinare l' ottima versione eschilea: è stato Euripide - per il motivo accennato sopra - a volerla modificare - o meglio ignorare, esattamente come Eschilo cinquant'anni prima aveva potuto ignorare le storie di Ares, Cefalo e Dedalo.

Il riesame critico dei testi qui proposto conferma la conclusione di Jacoby ${ }^{27}$ : non ci sono ragioni per togliere ad Eschilo la totalità dell'invenzione. Prima di Eschilo, gli unici imputati al processo areopagitico erano stati, secondo il mito attico, Ares, Cefalo e Dedalo. L'innovazione eschilea è destinata ad entrare in inevitabile contraddi-

25 Biehl (1965) 181. Così anche Chapouthier (1959) 100 n. 1 e West (1987) 292.

${ }^{26}$ Sommerstein (1989) 5.

27 Jacoby (1954a) 22-25, 42-43 e (1954b) 20-29, 47-49. Non fanno segnare progressi significativi rispetto a Jacoby le trattazioni di Gülke (1969) 25, Meier (1988) 149-253, Podlecki (1966) 88-89, Di Benedetto (1995) 128-129. Jacoby aveva avuto un predecessore più antico, uno sconosciuto critico di cui scrive Müller (1833) 157: „Daß Aischylos diese [scil. il processo areopagitico] erfunden habe, wie ein neuerer Gelehrter gemeint hat, ist durchaus unglaublich“. Alla identificazione di questo studioso hanno lavorato lo stesso Jacoby ed Eduard Fraenkel, ma invano: insieme al suo nome sono andate perdute le argomentazioni da lui avanzate che sarebbe stato estremamente interessante ricordare in questa sede.

[Rimane per me inspiegabile perché nella citazione, per il resto assolutamente fedele, che Jacoby (1954b) 24 n. 23 fa della frase di Müller si legga "neuerer“ invece di „heiterer“]. 
zione con questo mito. Per oscurarlo, Eschilo ha introdotto nel grande discorso di fondazione di Eum. 681-693 una nuova etimologia di Areopago, da lui stesso creata. In questi versi Atena annuncia che l'alta corte insediatasi sulla collina di Ares a celebrare per la prima volta un processo per delitto di sangue resterà in eterno a protezione della giustizia di Atene:

\begin{tabular}{|c|}
\hline 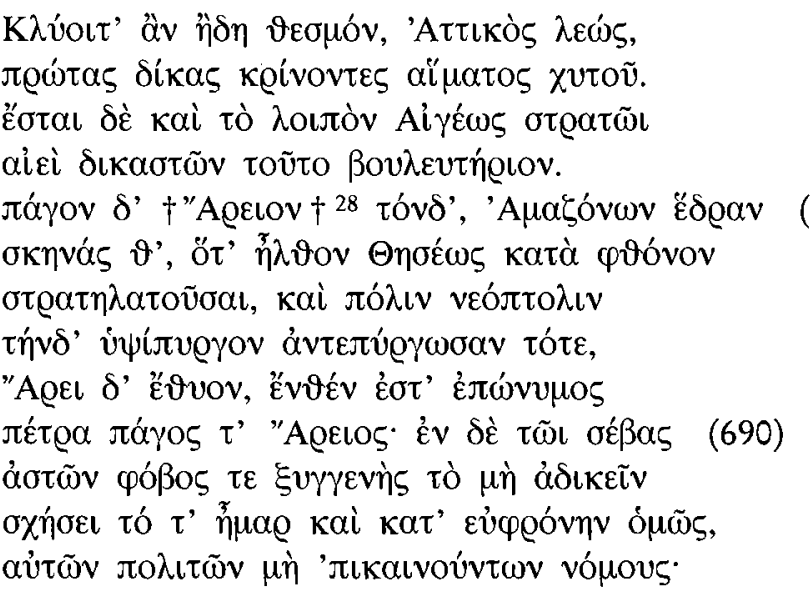 \\
\hline
\end{tabular}

La vecchia etimologia pre-eschilea è conservata ancora da Ellanico fr. 38 Fowler, proveniente dall'Atthis (pubblicata probabilmente dopo il 404-329):

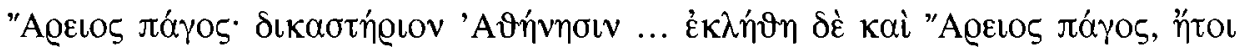

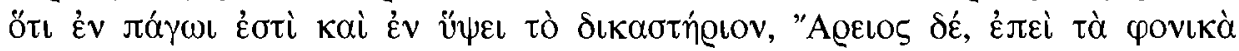

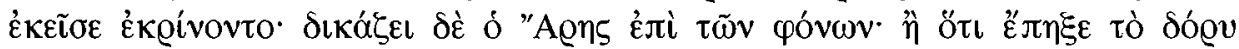

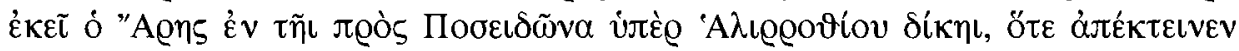

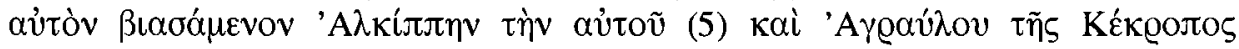

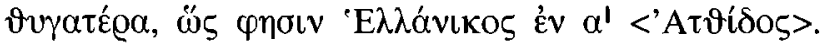

28 Accettare questo testo al v. 685 - come fanno Podlecki (1989) 104 e l'edizione Di Benedetto (1985) 528 significa ammettere un anacoluto, fenomeno per altro non raro in Eschilo. Si è però sospettata la genuinità di "A@elov, espunto da Page (1975) 273 e Sommerstein (1989) 66, 214. La difficoltà nel conservare il testo tradito consiste nel fatto che $\mathrm{i}$ due accusativi del v. 685 restano senza verbo reggente: esso, si è quindi supposto, potrebbe essere caduto ed essere stato sostituito proprio con "A@elov. Questa glossa, per dirla con Rose (1958) 275 , tradisce la mano di un intepolatore „troppo entusiasta“ che ha compreso ciò che verrà detto pochi versi dopo e lo vuole anticipare, rovinando così l'effetto del v. 690. Un criterio di scelta testuale potrebbe qui essere fornito da un corretto apprezzamento della strategia eschilea: il poeta voleva dare risalto alla nuova etimologia che stava proponendo e la strutturazione del passaggio che sarebbe servita meglio a questo scopo doveva far precedere la causa all'effetto: è il sacrificio ad Ares (e nient'altro che questo) che motiva il nome „Areopago“. Un'anticipazione di "Agelov al v. 685 avrebbe attenuato l'effetto di climax dell' affermazione. Per sanare il testo, congetture e.g. in Wecklein (1888) 299.

29 Per la datazione dell'Atthis di Ellanico cf. Jacoby (1954 a) 5-11. Questa etimologia doveva essere una costante nelle Atthides: si trovava anche in Apollodoro e Filocoro, per i quali cf. Jacoby (1904) 29. 
Nel discorso di fondazione di Atena Eschilo segue una via del tutto differente. Egli deve l'idea dell'accampamento sul colle di Ares non tanto alla tradizione quanto alla realtà storica del recente passato: l'Areopago era stato utilizzato come base militare dai Persiani. Che anche le Amazzoni avessero invaso l'Attica lo ricordava già un anonimo ateniese nelle storie di Erodoto (VIII 52,1). Ma (1) l'insediamento delle Amazzoni sull'Areopago e (2) il sacrificio lì compiuto delle Amazzoni ad Ares non sono ricordati da nessun altro autore; inventandoli, Eschilo ha ottenuto (1) la connessione tra il colle e le Amazzoni (sfruttando, probabilmente, un rinnovato interesse in materia di invasioni dopo quella persiana) e (2) il collegamento tra la Amazzoni e il nome Ares (Ares era già loro padre: cf. [Lys] II.4 ).

Creata così la nuova etimologia, Eschilo la inserì con un preciso proposito ${ }^{30}$ : voleva che gli spettatori dimenticassero quanto avevano sempre saputo (1) sui primi tre processi areopagitici e (2) sul fatto che l'Areopago era esistito dai tempi del re Cecrope e credessero invece (1) che il primo processo era quello di Oreste, cui anche loro avrebbero di lì a poco assistito, (2) che l'Areopago era di fondazione post-troiana e (3) che non aveva alcun rapporto col processo di Ares, processo che anzi non era mai avvenuto.

La nuova etimologia eschilea risponde bene alla concezione che ispira l'opera: inventata dal poeta, essa libera il colle dell'Areopago dalla presenza di Ares e lo rende disponibile per la risoluzione della vicenda di Oreste ${ }^{31}$. Se avesse riconosciuto l'esistenza di altri processi, Eschilo avrebbe anche riconosciuto che l'epocale mutamento nella concezione del diritto era già avvenuta ${ }^{32}$.

Fin qui per quanto concerne la "soppressione“ di Ares, Cefalo e Dedalo. Per quanto riguarda Oreste, non esisteva un voto sull'Areopago che Eschilo si sia limitato a trasformare in voto dell'Areopago: è venuta al poeta tragico, e a lui per primo, l'idea di sostituire una interminabile vendetta familiare con un giudizio legale.

Questa invenzione audace riuscì. Ellanico fu il primo non ateniese ad "accettare" il nuovo processo nelle sue 'Iég̨ıal. La domanda formulata all'inizio sul valore della testimonianza di Ellanico in relazione alla tradizione pre-eschilea può ricevere ora risposta: essa non ha alcun valore. Il fr. 169 Fowler si rivela molto più „eschileo" di

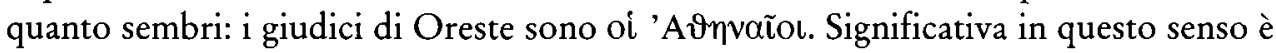
anche la struttura espositiva scelta da Ellanico: la cronologia relativa dei quattro processi è data non sulla base della lista dei re dell'Attica, ma a partire dal processo di

30 Altre, meno convincenti, spiegazioni in Blass (1906) 1-24 (tacendo di Ares, Eschilo avrebbe evitato di riferirsi a imbarazzanti litigi fra dei. Ma che dire della contesa tra Apollo e le Erinni?) e in Schmidt-Stählin (1934) 245 n. 8 (si rigetta la tradizione su Ares perché inconciliabile con la dignità di Poseidone. Ma come spiegare allora anche l'esclusione di Cefalo e Dedalo?).

31 La presenza di questa etimologia rende inoltre impossibile concludere che Eschilo non conosceva le altre saghe perché queste erano una tarda invenzione.

32 Questo era già stato osservato da Müller (1833) 157: „Den Areopag als schon bestehend zu setzen, würde Aeschylos Plänen wenig entsprochen haben“. 
Oreste risalendo all'indietro nel tempo. Ellanico sta cercando di sistemare (che vuol dire trovare un compromesso) il nuovo processo delle Eumenidi con i tre precedenti. Perché uno storico non può operare semplicemente rigettando le altre tradizioni più antiche, come invece aveva potuto fare il poeta tragico. Eschilo sfrutta appieno la libertà mitopoietica concessa al dramma: là dove la sorte dell'Oreste stesicoreo restava un fatto olimpico, egli coinvolge la coscienza civica ateniese, là dove Oreste era un singolo uomo salvato da un singolo atto di benevolenza di Apollo, Eschilo istituisce per lui e per Atene il tribunale areopagitico, vero $\kappa \tau \tilde{\eta} \mu \alpha \dot{\varepsilon} \zeta \alpha i \varepsilon i$.

Così l'Orestea si conclude con un istituto ateniese ed un omaggio ad Atene che risponde non solo alla ben nota contingenza storica in cui le Eumenidi furono composte ma anche all'idea di Giustizia eschilea.

\section{Bibliografia}

W. S. Barrett, Euripides. Hippolytos, Oxford 1964.

W. Biehl, Euripides Orestes, Berlin 1965.

F. Blass, Aischylos' Choephoren, Halle 1906.

W. Burkert, Homo Necans. Interpretationen altgriechischer Opferriten und Mythen, Berlin - New York, 2. Auflage 1996.

F. Chapouthier - L. Méridier, Euripides, Oreste, Paris 1959.

M. Croiset, Eschyle. Études sur l'invention dramatique dans son théatre, Paris 1928.

V. Di Benedetto, Euripidis Orestes, Firenze 1965.

-, (ed.) Eschilo. Orestea, Milano 1995.

R. L. Fowler, Early Greek Mythography, vol I: Text and introduction, Oxford 2000.

C. Gülke, Mythos und Zeitgeschichte bei Aischylos, Meisenheim am Glan 1969.

F. Jacoby, Das Marmor Parium, Berlin 1904.

-, Die Fragmente der griechischen Historiker I. Genealogie und Mythographie, Berlin 1923 (repr. Leiden 1957).

-, Die Fragmente der griechischen Historiker, Dritter Teil, b (Supplement) Nos. 323 a-334 Vol. I, Text, Leiden 1954 (cit. 1954 a).

-, Die Fragmente der griechischen Historiker, Dritter Teil, b (Supplement) Nos. 323 a-334 Vol. II, Notes, Leiden 1954 (cit. 1954b).

A. Lesky, Die Orestie des Aischylos, Hermes 66, 1931, 190-214 (= Gesammelte Schriften, Bern 1966, 92-111).

-, Orestes, RE XVIII 1, 1939, 966-1010.

P. Mazon, Eschyle (II), Paris 1955.

C. Meier, La nascita della categoria del politico in Grecia, Bologna 1988 ( $1^{\circ}$ ed. ted. Frankfurt am Main 1980).

K.O. Müller, Aeschylos. Eumeniden. Griechisch und Deutsch mit erläuternden Abhandlungen, Göttingen 1833.

D. L. Page, Aeschyli septem quae supersunt tragoediae, Oxford 1972.

R. Parker, Miasma. Pollution and Purification in early Greek Religion, Oxford 1983

M. Platnauer, Euripides. Iphigenia in Tauris, Oxford ${ }^{4} 1967$.

A. J. Podlecki, The Political Background of Aeschylean Tragedy, Ann Arbor 1966.

-, Aeschylus. Eumenides, Warminster 1989.

J.H. Quincey, Orestes and the Argive Alliance, CQ XIV, 1964, 190-206.

L. Radermacher, Das Jenseits im Mythos der Hellenen. Untersuchungen über Antiken Jenseitsglauben, Bonn 1903.

C. Robert, Die griechische Heldensage, 3. Buch, II. Abteilung, 2. Hälfte, Berlin 1926.

H.J. Rose, A Commentary on the Surviving Plays of Aeschylus, Amsterdam 1958. 
W. Schmidt - O. Stählin, Geschichte der griechischen Literatur, Erster Teil. Zweiter Band, München 1934.

A. H. Sommerstein, Aeschylus Eumenides, Cambridge 1989.

R. W. Wallace, The Areopagos Council, to 307 B. C., Baltimore 1985.

N. Wecklein, Äschylos' Orestie mit erklärenden Anmerkungen, Leipzig 1888.

O. Weinreich, Zwölfgötter, in: R. W. Roscher (Hrsg.), Ausführliches Lexicon der griechischen und römischen Mythologie, Vol. VI, Hildesheim - New York 21977, 764-848.

M. L. West, Euripides. Orestes, Warminster 1987.

-, Aeschyli Tragoediae cum incerti poetae Prometeo, Stuttgart 1990.

U. von Wilamowitz-Möllendorff, Die beiden Elektren, Hermes XVIII, 1883, 214-263

-, Griechische Tragödien übersetzt (II), Berlin 1904.

-, Aischylos Interpretationen, Berlin 1914.

C. W. Willink, Euripides. Orestes. Oxford, 1986.

\title{
Scuola Normale Superiore di Pisa
}

\section{I - 56100 Pis a}

\begin{abstract}
The importance of determining the exact origin of the trial of Orestes before the Areopagus at the end of Aeschylus's Eumenides has not been fully acknowledged by modern scholars. Through a close scrutiny of the surviving evidences concerning the genealogical book of Pherecydes, the aition of the Choes-festival and the roll of the Twelve Gods in the sphere of mythic history, this article suggests that there is no reason to accept the widespread belief that Aeschylus was (only) the heir of some pre-existent attic tradition. The trial of Orestes before the Athenian court turns out to be Aeschylus' own innovation, used by the poet to convey his new vision of justice.
\end{abstract}

